\title{
RuralCovidLife: Study protocol and description of the data
}

\section{[version 1; peer review: 1 not approved]}

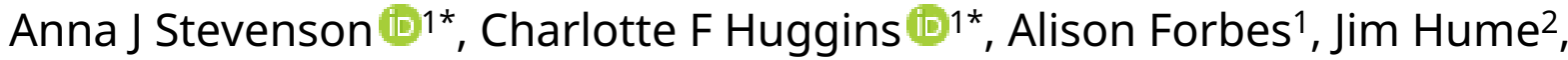 \\ Grant Fulton33, Claire Thirlwall4, Janet Miles (iD) 5, Chloe Fawns-Ritchie (i)1,6, \\ Archie Campbell(i)1,7, Clifford Nangle (iD7, Rebecca Dawson7, Rachel Edwards',8, \\ Robin Flaig (D)7, Louise Hartley (D)7, Christie Levein1, Daniel L McCartney1, \\ Ian J Deary (1D6, Caroline Hayward8, Riccardo E Marioni' ${ }^{1}$, Andrew M McIntosh (iD9, \\ Cathie Sudlow? ${ }^{7}$ David J Porteous (iD) 1,7
}

\footnotetext{
${ }^{1}$ Centre for Genomic and Experimental Medicine, Institute of Genetics and Cancer, University of Edinburgh, Edinburgh, EH4 2XU, UK

2Support in Mind Scotland, Edinburgh, EH16 5GA, UK

${ }^{3}$ Fulton Fisheries Consultancies Limited, Isle of Harris, HS3 3DX, UK

${ }^{4}$ Directorate of Public Health, Dumfries and Galloway Council, Dumfries, DG1 2DD, UK

${ }^{5}$ The GALE Centre, Gairloch, IV21 2BH, UK

${ }^{6}$ Department of Psychology, University of Edinburgh, Edinburgh, EH8 9JZ, UK

${ }^{7}$ Centre for Medical Informatics, Usher Institute, University of Edinburgh, Edinburgh, EH16 4UX, UK

${ }^{8}$ MRC Human Genetics Unit, Institute of Genetics and Cancer, University of Edinburgh, Edinburgh, EH4 2XU, UK

${ }^{9}$ Division of Psychiatry, University of Edinburgh, Edinburgh, EH10 5HF, UK

* Equal contributors
}

V1 First published: 23 Nov 2021, 6:317
https://doi.org/10.12688/wellcomeopenres.17325.1
Latest published: 23 May 2022, 6:317
https://doi.org/10.12688/wellcomeopenres.17325.2

\section{Abstract}

RuralCovidLife is part of Generation Scotland's CovidLife project, investigating the impact of the COVID-19 pandemic and mitigation measures on people in Scotland. The RuralCovidLife project focuses on Scotland's rural communities, and how they have been impacted by the pandemic.

During survey development, Generation Scotland consulted with people living or working in rural communities, and collaborated with a patient and public involvement and engagement (PPIE) group composed of rural community leaders. Through this consultation work, the RuralCovidLife survey was developed to assess the issues most pertinent to people in rural communities, such as mental health, employment, transport, connectivity, and local communities. Between 14th October and 30th November 2020, 3,365 participants from rural areas in Scotland took part in the survey. Participant ages ranged from 16 to 96 (mean $=58.4$, standard deviation $[S D]=13.3$ ), and the majority of the participants were female (70.5\%). Over half $(51.3 \%)$ had taken part in the original CovidLife survey.

RuralCovidLife includes a subsample $(n=523)$ of participants from the

\section{Open Peer Review}

Approval Status

1

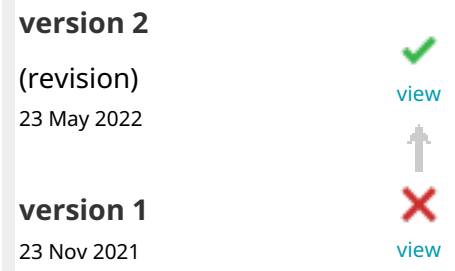

\footnotetext{
1. Holly Hope (iD), University of Manchester, Manchester, UK
}

Any reports and responses or comments on the article can be found at the end of the article. 
Generation Scotland cohort. Pre-pandemic data on health and lifestyle, as well as biological samples, are available for these participants. These participants' data can also be linked to past and future healthcare records, allowing analysis of retrospective and prospective health outcomes. Like Generation Scotland, RuralCovidLife is designed as a resource for researchers. RuralCovidLife data, as well as the linked Generation Scotland data, is available for use by external researchers following approval from the Generation Scotland Access Committee.

RuralCovidLife can be used to investigate mental health, well-being, and behaviour in participants living in rural areas during the COVID-19 pandemic, as well as comparisons with non-rural samples. Moreover, the sub-sample with full Generation Scotland data and linkage can be used to investigate the long-term health consequences of the COVID19 pandemic in rural communities.

Keywords

cohort, longitudinal study, COVID-19, rural communities

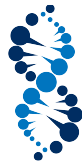

This article is included in the Generation

Scotland gateway.

Corresponding author: Charlotte F Huggins (chuggins@ed.ac.uk)

Author roles: Stevenson AJ: Data Curation, Formal Analysis, Visualization, Writing - Original Draft Preparation; Huggins CF: Data Curation, Visualization, Writing - Original Draft Preparation, Writing - Review \& Editing; Forbes A: Conceptualization, Investigation, Methodology, Project Administration; Hume J: Conceptualization, Writing - Review \& Editing; Fulton G: Conceptualization, Writing Review \& Editing; Thirlwall C: Conceptualization, Writing - Review \& Editing; Miles J: Conceptualization, Writing - Review \& Editing; Fawns-Ritchie C: Conceptualization, Investigation, Methodology, Writing - Review \& Editing; Campbell A: Conceptualization, Data Curation, Formal Analysis, Investigation, Methodology, Project Administration, Supervision, Writing - Review \& Editing; Nangle C: Conceptualization, Data Curation, Methodology, Project Administration; Dawson R: Conceptualization, Investigation, Project Administration; Edwards R: Conceptualization, Investigation, Methodology, Project Administration; Flaig R: Conceptualization, Project Administration, Supervision, Writing - Review \& Editing; Hartley L: Conceptualization, Methodology, Project Administration; Levein C: Project Administration; McCartney DL: Data Curation; Deary IJ: Conceptualization, Funding Acquisition, Supervision, Writing - Review \& Editing; Hayward C: Conceptualization, Funding Acquisition, Supervision; Marioni RE: Conceptualization; McIntosh AM:

Conceptualization, Funding Acquisition, Supervision; Sudlow C: Conceptualization, Funding Acquisition; Porteous DJ: Conceptualization, Funding Acquisition, Methodology, Project Administration, Supervision, Writing - Review \& Editing

Competing interests: Members of the PPIE panel involved in development of study also acted as co-authors.

Grant information: This work was supported by Wellcome [216767]; the Chief Scientist Office of the Scottish Government Health Directorates [CZD/16/6]; the Scottish Funding Council [HR03006]; the Wellcome Trust-University of Edinburgh Institutional Strategic Support Fund [ISSF3].

The funders had no role in study design, data collection and analysis, decision to publish, or preparation of the manuscript.

Copyright: @ 2021 Stevenson AJ et al. This is an open access article distributed under the terms of the Creative Commons Attribution License, which permits unrestricted use, distribution, and reproduction in any medium, provided the original work is properly cited.

How to cite this article: Stevenson AJ, Huggins CF, Forbes A et al. RuralCovidLife: Study protocol and description of the data [version 1; peer review: 1 not approved] Wellcome Open Research 2021, 6:317 https://doi.org/10.12688/wellcomeopenres.17325.1 First published: 23 Nov 2021, 6:317 https://doi.org/10.12688/wellcomeopenres.17325.1 


\section{Introduction}

The coronavirus (COVID-19) pandemic, and the resultant infection control measures, led to drastic changes in everyday life across the world. In the United Kingdom (UK), the first national lockdown was implemented on $23^{\text {rd }}$ March 2020, resulting in major restrictions to the population's way of living. This included workplace and school closures, strict instructions to stay at home except for very limited purposes, and the introduction of the Job Retention Scheme, placing many people in the UK on furlough for indeterminate periods of time. After the initial gradual easing of restrictions in 2020, a second lockdown was implemented in Scotland from $5^{\text {th }}$ January to $19^{\text {th }}$ July 2021.

From early in the pandemic, many have expressed concerns that such measures may have long-term consequences on mental health and well-being ${ }^{1}$, and that research is needed to document and measure these effects ${ }^{2}$. Subsequent longitudinal studies have found small increases in mental health difficulties during the COVID-19 pandemic compared to pre-pandemic ${ }^{3-8}$. However, effects are highly heterogeneous between studies, indicating that the impact of the pandemic may vary across populations ${ }^{5}$. For instance, young people and women tended to show greater negative effects during the pandemic compared to other groups $^{6-8}$. However, these studies did not closely examine differences between rural and urban settings.

In Scotland, $17 \%$ of the population live in rural locations ${ }^{9}$, classed as settlements with populations of fewer than 3,000 people. Moreover, rural communities typically face different challenges compared to the rest of the population, particularly in regards to transport, access to services, and employment. Despite this, rural communities have rarely been the focus of COVID-19 research.

The small body of existing evidence suggests that while rural communities may be similarly negatively affected by COVID-19 compared to urban communities, they also face unique challenges. Work on rural communities in the United States of America (USA) suggests the pandemic has had a strong negative effect on the well-being and economic status of people in these communities ${ }^{10}$. Moreover, the existing digital and health disparities between urban and rural communities may place rural residents at greater health risk from COVID-19 and the negative effects of the pandemic ${ }^{11}$. Research in rural China suggests that while rural residents were less likely to report psychological distress during the pandemic, they reported qualitatively different concerns, such as transport and digital $\operatorname{access}^{12}$.

These findings suggest that rural communities may face particular challenges during the COVID-19 pandemic. As such, a dataset capturing rural experiences during the pandemic would be valuable for future research, and for informing policy on rural recovery post-COVID-19. Generation Scotland developed the RuralCovidLife project to address this gap in available cohort data.
Generation Scotland is a large, longitudinal research study looking at the health and well-being of volunteers and their families across Scotland ${ }^{13}$. This cohort has detailed socio-demographic, psychological, and genetic data from over 24,000 volunteers across Scotland, collected between 2006 and 2011, as well as health record linkage ${ }^{13}$. From the beginning of the first national UK lockdown, Generation Scotland temporarily pivoted to conduct COVID-19 research.

In April 2020, Generation Scotland launched the CovidLife project, surveying how adults across Scotland were responding to the pandemic and subsequent COVID-19 mitigation measures ${ }^{14}$. Following the success of this project, two sister projects were launched: TeenCovidLife ${ }^{15}$, surveying Scottish adolescents, and RuralCovidLife, surveying people age 16 and above in rural Scotland. Rural Scotland is defined using the Scottish Government Urban-Rural Classification ${ }^{16}$, which classifies settlements with a population of less than 3,000 as rural.

RuralCovidLife was designed to assess how the COVID-19 pandemic and subsequent mitigation measures affected those living in Scottish rural communities. This was done through participatory work with people living and working in rural communities. The questions were designed by, and for, people from rural communities, to give a voice to those living in rural locations and influence policy defining how they will be supported in the future. The RuralCovidLife survey ran from 14th October to 30th November 2020 and was open to anyone aged 16 or over living in rural Scotland. This paper describes the development of the survey, characterises the cohort, and summarises the data available to researchers.

\section{Methods}

Questionnaire development

RuralCovidLife began with the goal of adapting the CovidLife surveys for use in rural communities. To do so, Generation Scotland consulted with members of rural communities, particularly industry and community leaders, as well as rural life experts from a range of specialities and areas. Participants in the consultation and patient and public involvement and engagement (PPIE) work included representatives from organisations such as the National Rural Mental Health Forum, Seafood Scotland, National Farmers Union Scotland, and the Scottish Rural Network.

This work informed the design of the RuralCovidLife survey. Informal consultations first identified the key challenges faced by rural communities in the COVID-19 pandemic. These key themes were discussed in the PPIE group to further refine the survey and ensure it was relevant to rural communities. The PPIE group also played an important role in promoting RuralCovidLife within their communities.

PPIE members shaped the topics addressed in the survey from the beginning of the project. They also helped refine the survey to be suitable for rural communities, providing feedback on the final questionnaire. Some PPIE members also helped to 
co-author the current paper, providing feedback and comments on the manuscript.

\section{Consultation}

Fourteen contacts took part in the initial consultations, all of whom were people living in rural Scottish communities and had expertise in relevant fields, such as tourism, business, or mental health. Initial informal consultations took place over the phone, during which informants were asked questions around the following topics:

- Key challenges faced by rural communities in lockdown

- Key challenges for rural communities leaving lockdown

- Main issues facing rural communities before lockdown

- The potential benefits of lockdown for rural communities

From these initial consultations, the following themes emerged as the main issues facing rural communities in the pandemic:

- Job loss and impact on the local economy

- Anxiety and frustration about tourism (e.g., re-opening of the Highlands while COVID-19 infections were still relatively high)

- Transport and accessibility

- Digital connectivity

- Under-representation of rural communities in both general and COVID-19 research and policy

- Different ways inequality manifests in rural communities compared to urban communities

- Having multiple jobs and mixed economy

- Mental health

The economic impact of lockdown emerged as one of the biggest issues for those interviewed, with many reporting that communities have been affected by changes to tourism and hospitality. Those interviewed believed that such changes could exacerbate pre-existing challenges to job security. Many of those interviewed also reported heightened anxiety in their communities with the easing of lockdown, particularly for young or elderly populations, with concerns that the re-opening of tourism would lead to higher rates of infection in rural areas. The consultation members also viewed existing issues around both private and public transport as another barrier to the recovery of rural communities post-lockdown, as well as a contributor to financial inequality.

The consultation also highlighted the resilience of rural communities. Many interviewed felt local rural communities were faster in providing necessary support than the government or local councils, indicating that rural communities may not rely on government structures to the same extent as an urban community. Moreover, consultants viewed this sense of independence and unity as a key strength of rural communities and claimed that such strength will be essential for recovering from the impact of the pandemic and associated mitigation measures.
This consultation directly informed how to adapt the CovidLife surveys for rural participants, as well as what topics needed to be introduced to reflect the priorities of rural communities in Scotland.

\section{Public and patient involvement and engagement groups}

A key part of the development of the RuralCovidLife survey was the creation of a PPIE group, and running workshops with this group. The group brought together the viewpoints and experiences of people living or working in rural communities in Scotland. This PPIE group was set up remotely and all sessions were run online due to the COVID-19 restrictions. Seven people from across Scotland took part in this PPIE group, all of whom lived in rural communities, worked in rural areas, or both.

To build rapport before the workshops, each member of the PPIE group had at least two individual calls with a member of the Generation Scotland team. These calls built trust with group members and ensured all members fully understood what their participation would involve. The group took part in several online workshops, either one-to-one with a member of the Generation Scotland team, or paired with another PPIE group member. Workshops were conducted via online video conferencing or over the phone. The format for each session was chosen based on the group member's availability, preference, and internet access.

In these workshops, participants read the drafted questions for the RuralCovidLife survey, discussing the questionnaire and providing feedback to ensure questions authentically represented the priorities of rural communities and were inclusive to members of those communities.

Regular contact was maintained with the PPIE group, sharing information about the launch of the survey, press coverage, engagement levels, and other relevant information. They also received the initial report ${ }^{17}$ before it was shared with the press and published online. All members were also offered to be authors on the current paper, or to be included as a named acknowledgement. The PPIE members who elected to be part of the author team and read, commented on and approved the manuscript, were included in the author list.

\section{Building the questionnaire}

The RuralCovidLife questionnaire was developed by the Generation Scotland team using Qualtrics survey software ${ }^{18}$ and could be completed on desktop computers, tablets, and smartphones. Due to COVID-19 restrictions, data collection for RuralCovidLife was limited to online assessments only.

Due to the potentially sensitive nature of some questions in the survey, answering each question was not compulsory. Additionally, participants could choose 'Prefer not to answer' for many questions. If a participant left a question unanswered, they were informed of the missing response and asked to confirm if they wanted to continue without answering. Participants could save their responses and return to the survey 
later to complete them. The survey took approximately 30 minutes to complete.

\section{Sample and recruitment}

Anyone age 16 or over and living in rural Scotland was eligible to take part in the RuralCovidLife survey. While 'rural Scotland' was not defined in any further detail in the consent material, postcode was collected so that participant's residence could be compared to the Scottish Rural-Urban Classification?.

Internet access was required to participate. Data collection commenced on Wednesday $14^{\text {th }}$ October 2020 and closed to new participants on Monday $30^{\text {th }}$ November. Any participant who had started, but not finished the survey, had 14 days to complete their responses.

Various recruitment strategies were used to enroll participants into RuralCovidLife. Email invitations were sent to 5,080 eligible participants from CovidLife, TeenCovidLife, and Generation Scotland cohort members with rural postcodes. Of these, 470 were undeliverable. Those invited who had not yet completed the survey or taken part were sent reminder emails on the $16^{\text {th }}$ November 2020. Of the 4,610 invited, $46.9 \%(n=2,163)$ responded and were included in the final dataset.

The National Rural Mental Health Forum helped launch the RuralCovidLife survey via its 200 membership organisations by holding an online seminar on October $14^{\text {th }} 2020$. Attendees were from the private, public and third sector, and national media promoted the launch.
The PPIE group also played a crucial role in promoting the survey within their communities, with a number taking part in $\mathrm{TV}$, radio, and print media interviews at launch. Information about taking part in the study was also included in the newsletters and social media of industry networks in rural Scotland, including Scottish Rural Action, the Crofting Federation, the National Farmers' Union of Scotland, and the Rural Youth Project.

Geo-targeted social media advertising targeted participants from rural areas in Scotland, areas under-represented in wider research and in the initial survey response, such as the North West and the Scottish borders. This includes both organic and paid-for social media.

Participants were also recruited through the Scottish Health Research Register (SHARE) ${ }^{19}$. SHARE is a register of people age 11 and over in Scotland who expressed an interest in taking part in health research. After selecting those with rural postcodes, 7,190 members of their database were invited to take part.

\section{Questionnaire content}

The RuralCovidLife questionnaire content was developed to capture how the pandemic had impacted those living in rural communities. A summary of the topics assessed in the survey is shown in Table 1. The full RuralCovidLife questionnaire is available in the Extended data ${ }^{20}$. The Qualtrics survey file can also be requested from the authors.

\begin{tabular}{|c|c|}
\hline Sociodemographic & $\begin{array}{l}\text { Age; sex; gender identity; ethnic origin; postcode; household composition; relationship } \\
\text { status; household income; receipt of benefits; highest educational qualification; } \\
\text { accommodation type and tenure; dependents in household; caring responsibilities }\end{array}$ \\
\hline Health & $\begin{array}{l}\text { Self-reported health conditions; whether contacted about shielding; whether had COVID-19 } \\
\text { (suspected or tested); COVID-19 symptoms; healthcare access }\end{array}$ \\
\hline Psychological & Depressive symptoms; anxiety symptoms; wellbeing; life satisfaction \\
\hline $\begin{array}{l}\text { Social support and } \\
\text { relationships }\end{array}$ & Loneliness; isolation; whether someone could provide support if had COVID-19 \\
\hline Employment and finances & $\begin{array}{l}\text { Employment status (prior to COVID-19 pandemic and now); employment industry; opinions } \\
\text { on industries impacted by COVID-19 pandemic; whether furloughed; whether working } \\
\text { from home; key worker status; concern about impact of COVID-19 on business/livelihood; } \\
\text { application for support for business/livelihood; job security; financial situation (prior to } \\
\text { COVID-19 pandemic and now); concern about finances }\end{array}$ \\
\hline $\begin{array}{l}\text { COVID-19 knowledge, } \\
\text { attitudes and behaviour }\end{array}$ & $\begin{array}{l}\text { Confidence in Scottish government to prevent further outbreaks of COVID-19; attitudes } \\
\text { towards government guidelines for COVID-19; whether following government guidelines for } \\
\text { COVID-19; whether or not installed the Protect Scotland (NHS Scotland Test \& Protect) app }\end{array}$ \\
\hline Connectivity & $\begin{array}{l}\text { Internet connection type and quality; whether applied for Scottish Broadband Voucher } \\
\text { Scheme; importance of reliable high speed broadband for work, keeping in touch with } \\
\text { friends and family, accessing health and support services, and children's school work }\end{array}$ \\
\hline Transport and accessibility & $\begin{array}{l}\text { Whether consider where they live to be rural; whether live on island; public transport } \\
\text { use (prior to COVID-19 pandemic and now); difficulty planning routes (prior to COVID-19 } \\
\text { pandemic and now); vehicle ownership; whether need to drive as part of job }\end{array}$ \\
\hline Community & $\begin{array}{l}\text { Rating of local area as place to live; feeling of belonging; community engagement (prior } \\
\text { to COVID-19 pandemic and now); concern about community knowing about COVID-19 } \\
\text { infection; how aspects of community life have been affected by COVID-19 measures; } \\
\text { concern about visitors; attitudes on when tourists should be allowed to return }\end{array}$ \\
\hline
\end{tabular}


Psychological distress was measured with commonly used, validated scales. The nine-item Patient Health Questionnaire (PHQ-9) was used to assess depression ${ }^{21}$, and anxiety symptoms were assessed with the seven-item Generalised Anxiety Disorder (GAD-7) ${ }^{22}$. Loneliness, social isolation, and life satisfaction were also assessed. Other questions on topics such as transport and connectivity were drawn from the longitudinal Scottish Household Questionnaire 2018 23 , as well as cross-sectional studies such as the National Rural Mental Health Survey Scotland ${ }^{24}$, and the Scottish Rural Action Covid Survey $^{25}$.

\section{Procedure}

A personalised link to the RuralCovidLife survey was included in the email invitations, and a general link was shared on social media for the general public. The survey began with a participant information sheet outlining the aims of the survey and what involvement would entail. Following this, participants completed a consent form confirming their age, rural location, and consent to re-contact in the future. The consent and volunteer information sheet is available in the Extended data $^{20}$. After beginning the survey, participants had 14 days to complete it. They could stop and return to the survey at any point during this time.

\section{Ethical considerations}

RuralCovidLife was reviewed and given $\mathrm{s}$ favourable opinion by the East of Scotland Research Ethics Committee (Reference: 20/ES/0021 AM09).

\section{Results}

Between $14^{\text {th }}$ October and $30^{\text {th }}$ November 2020, data was collected for RuralCovidLife in Qualtrics. Two surveys were created to gather data. The first was available to the general public and could be accessed by anyone. The second was distributed directly to invited participants from Generation Scotland or CovidLife, using personal links that allowed linkage to their GS or CovidLife data. Both the general public and linked versions of the questionnaires can be seen in the Extended data ${ }^{20}$

In total, 4,635 responses were recorded. Responses were recorded even if participants only opened the survey, or did not answer any of the questions.

Data cleaning was conducted separately by two researchers. Final exclusions were compared and any inconsistencies were resolved through discussion and further analysis until exclusions were identical. Criteria for inclusion are as follows:

1. Participants complete the survey after the official launch (Excluded $\mathrm{n}=5$ ).

2. Participants must have submitted at least one page of the survey after completing the consent procedure (Excluded $\mathrm{n}=1,224)$.

3. Participants took part in the survey only once. Participants were classed as duplicates if both their names and email addresses were exactly the same. If email addresses were identical but names differed even slightly, they were classed as separate participants using a shared email address. For records from duplicate participants, the most completed record was preserved. If records had the same amount of progress through the questionnaire, the first record was taken (Excluded $n=34)$.

4. Participants must have answered at least one question across the survey (i.e., data must not have consisted of NA responses only; (Excluded $\mathrm{n}=7$ ).

Following data cleaning, 1,270 participants were included, leaving 3,365 participants in the final dataset.

Figure 1 shows the completion dates of the survey. Over half of these participants had additionally taken part in the original CovidLife survey $(\mathrm{n}=1,744,51.8 \%)$ and $523(15.5 \%)$ participants were members of Generation Scotland. A $0.7 \%$ $(n=23)$ fraction also took part in TeenCovidLife.

Scottish rural-urban classification was derived from participant postcodes, based on the Scottish Government Rural-Urban Classification". Some participants $(4.4 \% ; n=149)$ were classed as living in a non-rural area, despite the survey requiring self-selection of people from rural areas. These participants were retained in the dataset as all participants confirmed their rural location in the consent, and thus their given postcode may not reflect their involvement in rural life (e.g., those who may have been temporarily relocated during data collection). However, these participants can be easily excluded from future analyses if necessary.

Demographic information for these participants is shown in Table 2. The sample comprised of 2,372 female participants $(70.5 \%)$ and 979 male participants $(29.1 \%)$, ranging in age from 16 to 96 years $($ mean $=58.4$, standard deviation $[S D]=13.3)$. The age distribution of participants by sex is shown in Figure 2.

The majority of the participants were white $(n=3,137,93.2 \%)$ and lived in less deprived areas (Scottish Index of Multiple Deprivation [SIMD] decile $>5: \mathrm{n}=2,696,80.1 \%)$. Almost half were educated to degree level $(n=1,667,49.5 \%)$.

A total of $7.0 \%(n=235)$ had been told they were at severe risk from COVID-19 and needed to shield themselves. A tenth of participants $(10.4 \% ; n=349)$ reported that they had or had had COVID-19, whether suspected or confirmed with a test.

Table 3 shows the number of participants by local authority area. The highest proportion of participants were from Aberdeenshire $(\mathrm{n}=542,16.1 \%)$; The Aberdeenshire area, the Perth and Kinross area, and the Highland area accounted for $42.3 \%$ of participants.

Postcodes were mapped onto the Scottish Government Rural-Urban classification"; most participants lived in accessible 


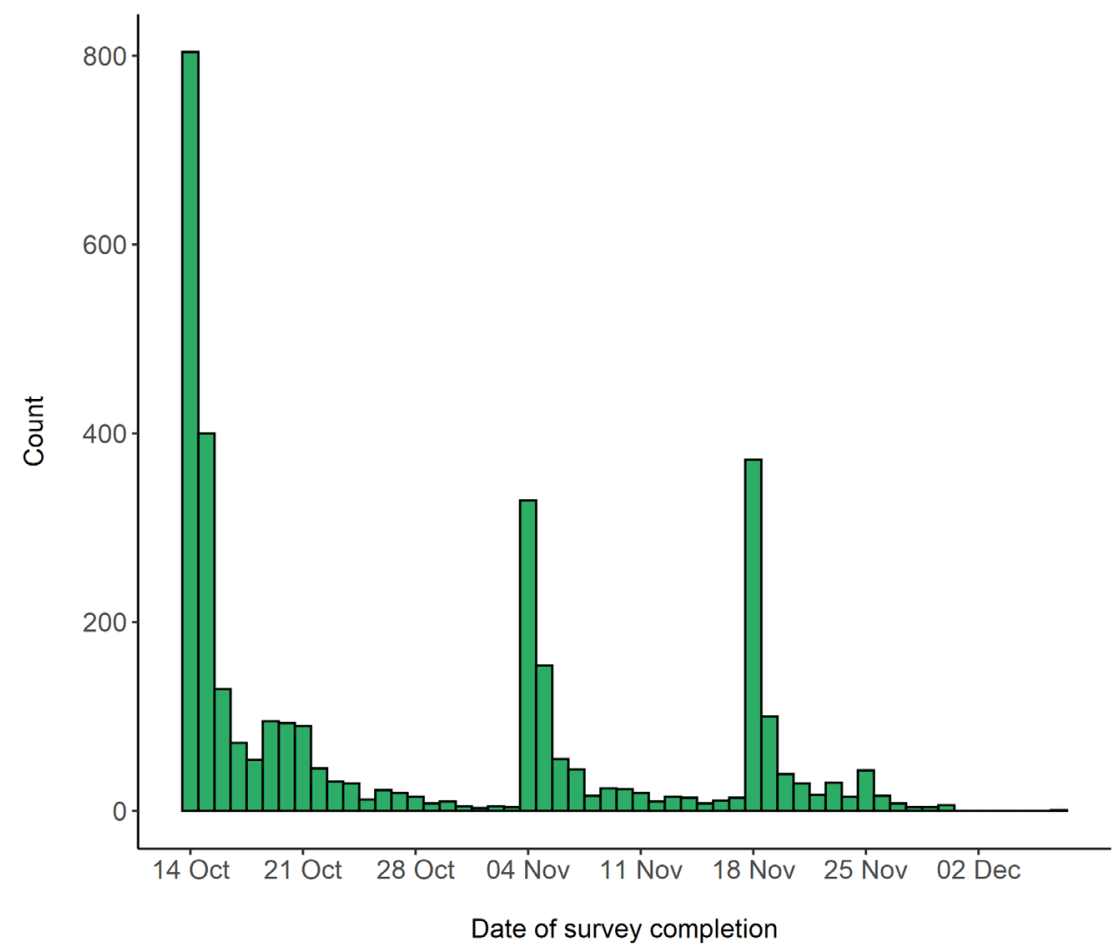

Figure 1. Survey completion dates for RuralCovidLife.

Table 2. Demographic characteristics of the RuralCovidLife sample.

\begin{tabular}{|l|c|}
\hline & Mean (SD) \\
\hline Age (years) & $58.4(13.3)$ \\
\hline Sex & (\%) \\
\hline Female & $2,372(70.5)$ \\
\hline Male & $979(29.1)$ \\
\hline Gender & \\
\hline Female & $2,364(70.2)$ \\
\hline Male & $975(29.0)$ \\
\hline Urban/rural classification & \\
\hline Remote rural & $1,054(31.3)$ \\
\hline Accessible rural & $2,099(62.4)$ \\
\hline Remote small towns & $52(1.5)$ \\
\hline Non-rural & $149(4.4)$ \\
\hline Data unavailable & $11(0.3)$ \\
\hline Ethnicity & $25(0.7)$ \\
\hline White & $27(0.8)$ \\
\hline Non-White & $176(5.2)$ \\
\hline Prefer not to answer & \\
\hline No response & \\
\hline
\end{tabular}

\begin{tabular}{|c|c|}
\hline & Mean (SD) \\
\hline Education & \\
\hline Degree & $1,667(49.5)$ \\
\hline No degree & $1,485(44.1)$ \\
\hline Prefer not to answer & $27(0.8)$ \\
\hline No response & $186(5.5)$ \\
\hline SIMD deciles & \\
\hline 1 - 2 (most deprived) & $20(0.6)$ \\
\hline $3-4$ & $255(7.6)$ \\
\hline $5-6$ & $1,060(31.5)$ \\
\hline 7 - 8 & $1,576(46.8)$ \\
\hline $9-10$ (least deprived) & $442(13.1)$ \\
\hline
\end{tabular}

SD: standard deviation; SIMD: Scottish Index of Multiple Deprivation

Data on Rural-Urban classification for participants who did not provide a valid postcode rural locations $(\mathrm{n}=2,099,62.4 \%)$, with $31.3 \%(\mathrm{n}=1,054)$ living in remote rural, and $1.5 \%(n=52)$ living in remote small towns. A subgroup of the sample lived on one of the Scottish islands $(\mathrm{n}=290,8.6 \%)$.

Of the total sample, 91\% $(\mathrm{n}=3058)$ had full GAD-7 data. Of these, $17.4 \%(\mathrm{n}=531)$ scored 10 or above $^{22}$, evidencing potential clinical anxiety. The median score was $3(\mathrm{IQR}=7)$. 


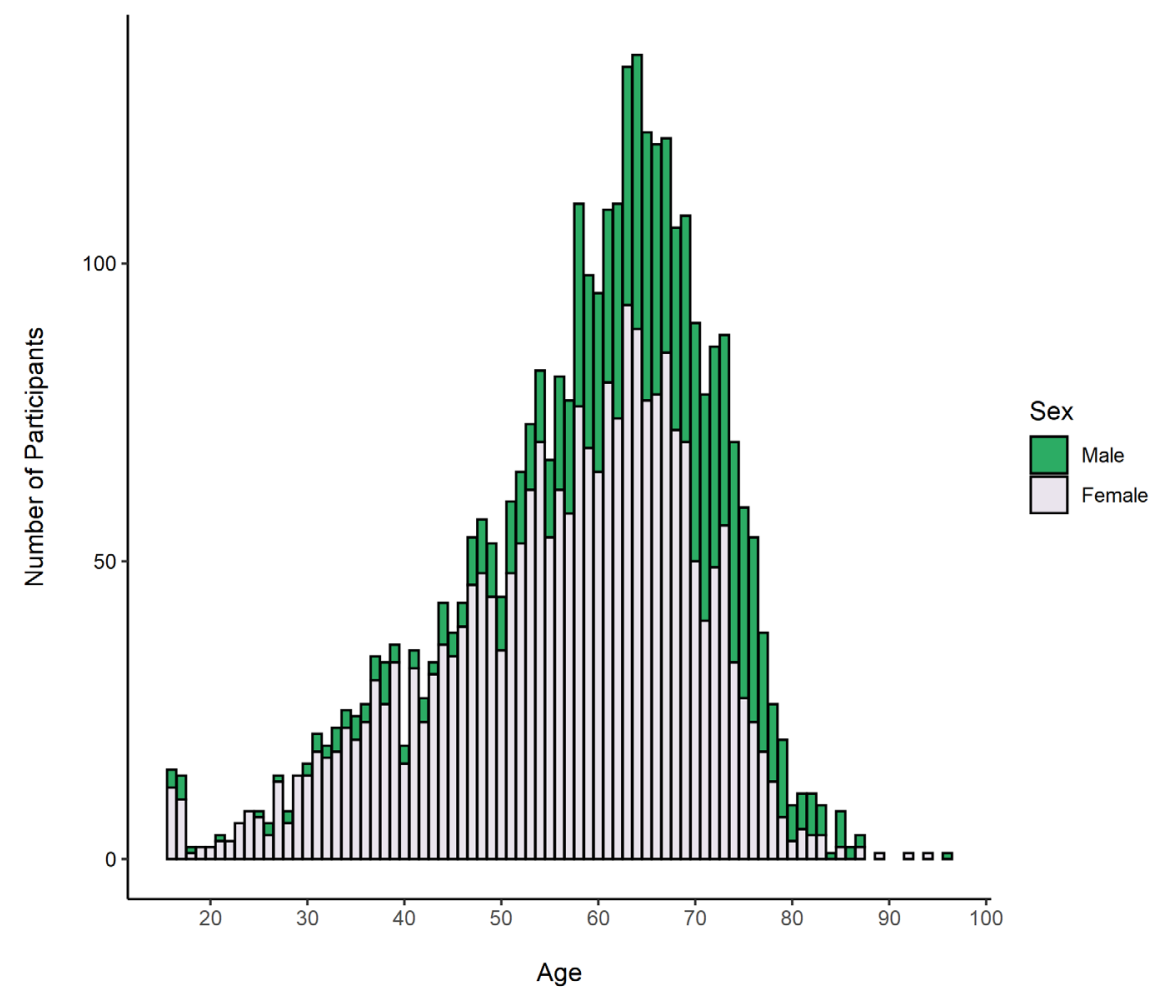

Figure 2. Age distribution of RuralCovidLife participants grouped by sex.

\begin{tabular}{|l|c|}
\hline $\begin{array}{l}\text { Table 3. Responses by local } \\
\text { authority areas. }\end{array}$ \\
\hline Local authority & $22(0.7)$ \\
\hline Aberdeen city & $542(16.1)$ \\
\hline Aberdeenshire & $292(8.7)$ \\
\hline Angus & $158(4.7)$ \\
\hline Argyll and Bute & $17(0.5)$ \\
\hline City of Edinburgh & $20(0.6)$ \\
\hline Clackmannanshire & $224(6.7)$ \\
\hline Dumfries and Galloway & $<10$ \\
\hline Dundee City & $22(0.6)$ \\
\hline East Ayrshire & $23(0.7)$ \\
\hline East Dunbartonshire & $95(2.8)$ \\
\hline East Lothian & $<10$ \\
\hline East Renfrewshire & $17(0.5)$ \\
\hline Falkirk & $332(9.9)$ \\
\hline Fife & $<10$ \\
\hline Glasgow City & $368(10.9)$ \\
\hline Highland & $37(1.1)$ \\
\hline Midlothian & \\
\hline
\end{tabular}

\begin{tabular}{|l|c|}
\hline Local authority & N (\%) \\
\hline Moray & $83(2.5)$ \\
\hline Na h-Eileanan Siar & $55(1.6)$ \\
\hline North Ayrshire & $28(0.8)$ \\
\hline North Lanarkshire & $18(0.5)$ \\
\hline Orkney Islands & $55(1.6)$ \\
\hline Perth and Kinross & $515(15.3)$ \\
\hline Renfrewshire & $17(0.5)$ \\
\hline Scottish Borders & $133(4.0)$ \\
\hline Shetland Islands & $53(1.6)$ \\
\hline South Ayrshire & $33(1.0)$ \\
\hline South Lanarkshire & $88(2.6)$ \\
\hline Stirling & $60(1.8)$ \\
\hline West Dunbartonshire & $<10$ \\
\hline West Lothian & $35(1.0)$ \\
\hline
\end{tabular}

Of the total sample, 88\% $(n=2973)$ had full PHQ-9 data. Of these, $19.2 \%(n=571)$ scored 10 or above ${ }^{26}$, evidencing potential clinical depression. The median score was $3(\mathrm{IQR}=7)$.

Figure 3 shows distribution of GAD-7 and PHQ-9 scores across participants. 

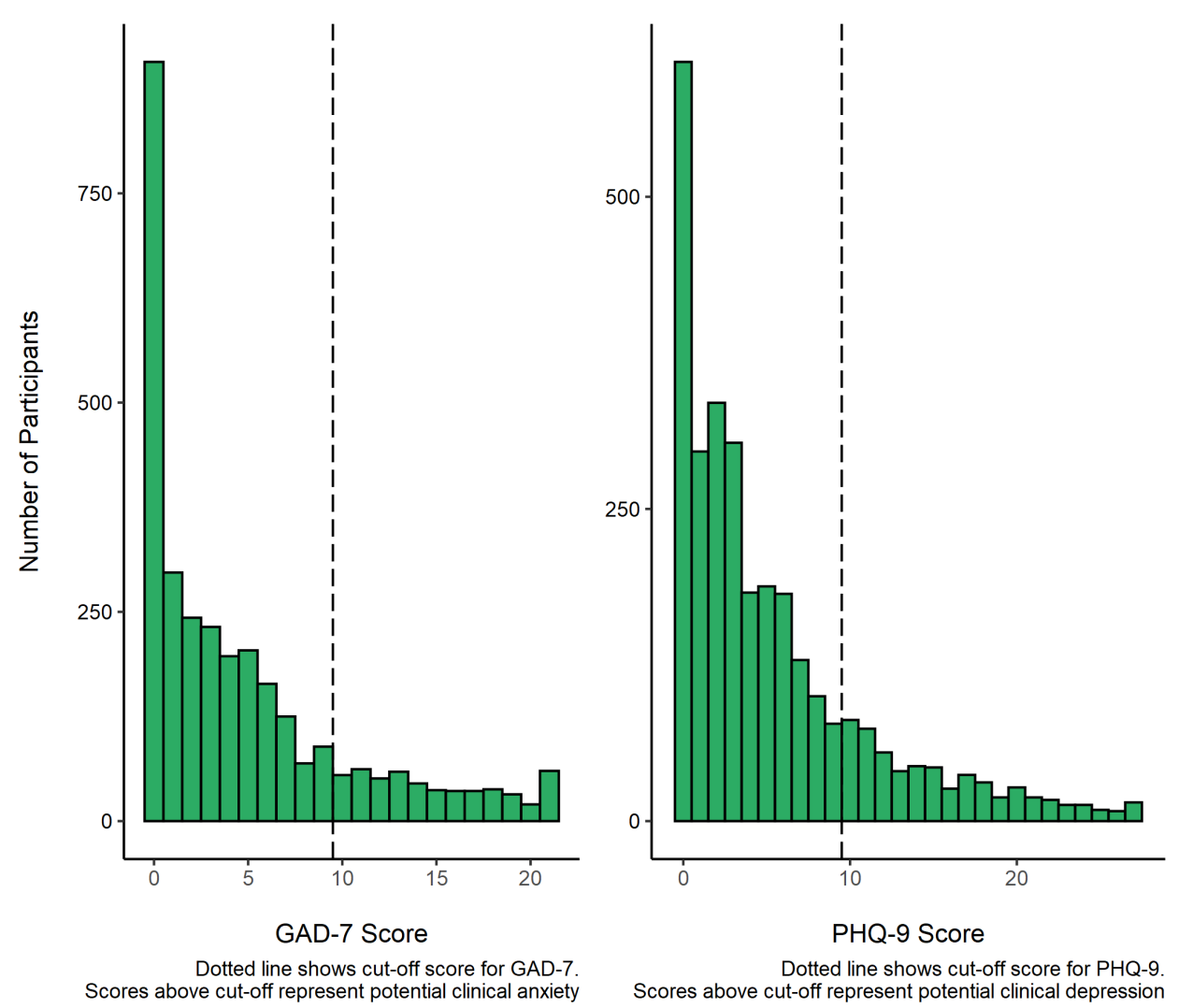

Figure 3. GAD-7 and PHQ-9 scores in RuralCovidLife participants.

Overall, $8.1 \%(n=264)$ of participants reported feeling lonely most or all of the time in the past week. Figure 4 shows the percentage of participants reporting frequency of loneliness by age group, after excluding participants who did not answer the question or responded 'Prefer not to say' or 'Don't know' ( $\mathrm{n}=106,3.2 \%$ ).

Participants rated overall life satisfaction from 0 ('Not at all satisfied') to 10 ('Extremely satisfied'), with 5 representing 'Neither satisfied or dissatisfied'. Mean life satisfaction score was $6.14(\mathrm{SD}=2.32)$, and 22.9\% $(\mathrm{n}=742)$ participants reported low life satisfaction.

Early results were reported and distributed online ${ }^{17}$, and can be accessed for free on the Generation Scotland website.

\section{Strengths and limitations}

The RuralCovidLife study offers a unique insight into how rural Scottish communities specifically were impacted by the COVID-19 pandemic from the first lockdown in March to autumn 2020. Through individual and small group consultations with people with lived experience in rural communities, the survey incorporated the issues most pertinent to rural Scotland in its design. The resulting dataset is one of the largest COVID-19 studies of the rural population in Scotland. Studying how these communities were differentially affected by the pandemic may be important to implement support in the future. Furthermore, the dataset captures information from a wide range of ages and locations across Scotland.

As RuralCovidLife took advantage of existing research projects within Generation Scotland, different datasets can be linked together. In particular, 523 RuralCovidLife participants were Generation Scotland volunteers and had extensive pre-pandemic data available. This makes it possible to link this subset of RuralCovidLife data not only to pre-pandemic survey data but also, for example, to genetic and health record data. Moreover, through continued health-record linkage in Generation Scotland, it is also possible to examine the prospective impact of the pandemic on health and wellbeing for this subsample. Responses to RuralCovidLife can also be linked to CovidLife data for any participants who took part in both projects, allowing comparison to initial pandemic data in April 2020.

Due to COVID-19 restrictions, the study was conducted remotely and was thus restricted to those with Internet access, which may exclude at-risk groups with poor digital connectivity. 


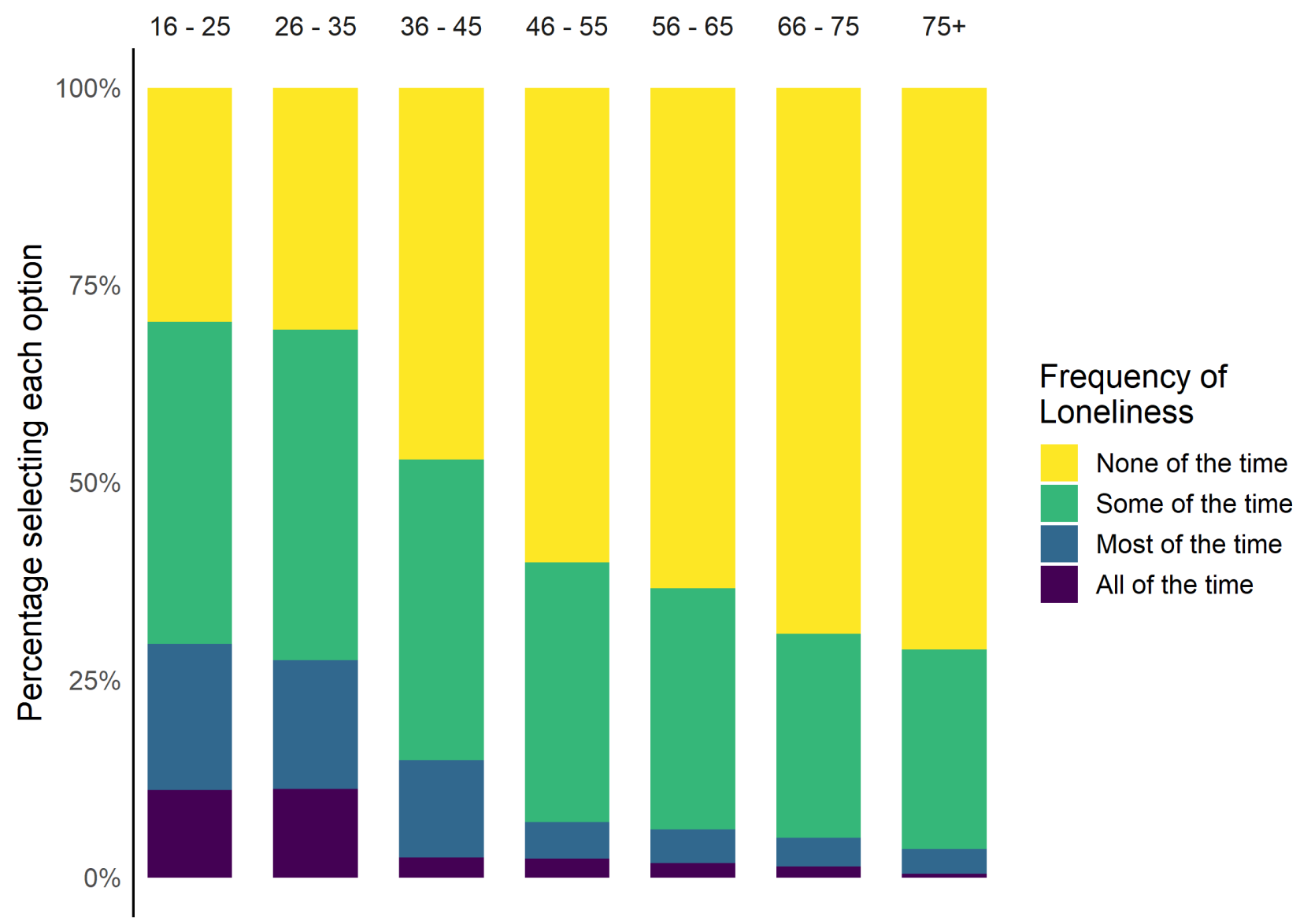

Age Group

Figure 4. Percentage of participants reporting different frequencies of loneliness.

However, this may also have allowed participants from more remote areas who would otherwise struggle to participate in research projects to get involved. Additionally, the study is not representative, with the majority of respondents being older and female. Participants were recruited through a mental health network, and a high proportion of participants scored above cut-off on the GAD-7 and PHQ-9. As such, participants with mental distress may be over-represented. Very few ethnic minority participants took part, making comparisons based on ethnic background unlikely to be tenable. Finally, recruitment was largely reliant on people who have already expressed an interest in health research, through participation in projects such as SHARE or Generation Scotland. These factors may limit the generalisability of the data.

The data was collected at a relatively early stage of the pandemic, before any COVID-19 vaccines became available. As such, this dataset does not capture uptake or attitudes towards COVID-19 vaccines. However, vaccine uptake in the sub-sample who are also part of Generation Scotland (15.6\%) can be assessed through examining linked data.

\section{Conclusions}

RuralCovidLife provides a valuable resource for investigating the effects of the COVID-19 pandemic on people in rural Scotland, built from a collaboration between researchers and members of those communities. While the pandemic has been challenging for many people, the remoteness of rural areas and their reliance on tourism means that rural communities face particular challenges. RuralCovidLife provides data to learn more about how rural communities have responded to the pandemic, and to help these communities recover in the future.

\section{Data availability}

Underlying data

The de-identified RuralCovidLife dataset is available to researchers in the UK and internationally. Researchers can apply for 
access to the RuralCovidLife data on the Generation Scotland website. The application form and further details of how to apply can be found at on the Generation Scotland website.

\section{Extended data}

Zenodo: Extended data for "RuralCovidLife: Study protocol and description of the data", https://doi.org/10.5281/ zenodo. $5589038^{20}$

This project contains the following extended data:

- 2021-10-16 RuralCovidLife Questionnaire GENERAL PUBLIC.docx

- 2021-10-16 RuralCovidlife Questionnaire PERSONALISED LINK.docx

- 2021-10-16 RuralCovidLife VIS \& Consent.docx

- CovidLife_Access_Request_Form_V3.1_March _2021. docx
- Generation_Scotland_Access_Request_Form_V1.2_March_ 2021.docx

Data are available under the terms of the Creative Commons Attribution 4.0 International license (CC-BY 4.0).

\section{Acknowledgements}

We thank all the participants who took part in the RuralCovidLife study. We would particularly like to thank the members of the PPIE group who informed this work, including Rhoda Meek, Kate Thomson, Fiona Thompson, and JC.

Recruitment to this study was facilitated by SHARE - the Scottish Health Research Register and Biobank. SHARE is supported by NHS Research Scotland, the Universities of Scotland, and the Chief Scientist Office of the Scottish Government.

\section{References}

1. Keep mental health in mind. Nat Med. 2020; 26(5): 631 PubMed Abstract | Publisher Full Text | Free Full Text

2. Holmes EA, O'Connor RC, Perry VH, et al.: Multidisciplinary research priorities for the COVID-19 pandemic: a call for action for mental health science. Lancet Psychiat. 2020; 7(6): 547-560.

PubMed Abstract | Publisher Full Text | Free Full Text

3. Pierce $M$, Hope $H$, Ford $T$, et al:: Mental health before and during the COVID-19 pandemic: a longitudinal probability sample survey of the UK population. Lancet Psychiat. 2020; 7(10): 883-892. PubMed Abstract | Publisher Full Text | Free Full Text

4. Robinson $E$, Sutin $A R$, Daly $M$, et al.: A systematic review and meta-analysis of longitudinal cohort studies comparing mental health before versus during the COVID-19 pandemic. medRxiv. 2021; 21252921. Publisher Full Text

5. Prati G, Mancini AD: The psychological impact of COVID-19 pandemic lockdowns: a review and meta-analysis of longitudinal studies and natural experiments. Psychol Med. 2021; 51(2): 201-211.

PubMed Abstract | Publisher Full Text | Free Full Text

6. Niedzwiedz CL, Green MJ, Benzeval M, et al.: Mental health and health behaviours before and during the initial phase of the COVID-19 lockdown: longitudinal analyses of the UK Household Longitudinal Study. J Epidemiol Community Health. 2021; 75(3): 224-231.

PubMed Abstract | Publisher Full Text | Free Full Text

7. Daly M, Sutin AR, Robinson E: Longitudinal changes in mental health and the COVID-19 pandemic: evidence from the UK Household Longitudinal Study. Psychol Med. 2020; 1-10

PubMed Abstract | Publisher Full Text | Free Full Text

8. Kwong ASF, Pearson RM, Adams MJ, et al.: Mental health during the COVID-19 pandemic in two longitudinal UK population cohorts. medRxiv. 2020; 20133116

Publisher Full Text

9. Scottish Government: Rural Scotland Key Facts 2021. 2021. Reference Source

10. Mueller JT, McConnell K, Burow PB, et al.: Impacts of the CoviD-19 pandemic on rural America. Proc Natl Acad Sci U S A. 2021; 118(1): 2019378118. PubMed Abstract | Publisher Full Text | Free Full Text

11. Summers-Gabr NM: Rural-urban mental health disparities in the United States during COVID-19. Psychol Trauma. 2020; 12(S1): S222-S224. PubMed Abstract | Publisher Full Text

12. Liu L, Xue $P$, Li SX, et al.: Urban-rural disparities in mental health problems related to COVID-19 in China. Gen hosp psychiat. 2021; 69: 119-120. PubMed Abstract | Publisher Full Text | Free Full Text

13. Smith BH, Campbell A, Linksted P, et al:: Cohort Profile: Generation Scotland: Scottish Family Health Study (GS:SFHS). The study, its participants and their potential for genetic research on health and illness. Int J Epidemiol. 2013; 42(3): 689-700.

PubMed Abstract | Publisher Full Text

14. Fawns-Ritchie C, Altschul DM, Campbell A, et al:: CovidLife: a resource to understand mental health, well-being and behaviour during the COVID-19 pandemic in the UK [version 1; peer review: 1 approved] . Wellcome Open Research. 2021; 6: 176. Publisher Full Text

15. Huggins CF, Fawns-Ritchie C, Altschul DM, et al:: TeenCovidLife: a resource to understand the impact of the COVID-19 pandemic on adolescents in Scotland [version 1; peer review: awaiting peer review]. Wellcome Open Research. 2021; 6: 277 Publisher Full Text

16. Scottish Government: Rural Scotland Key Facts 2021. Edinburgh, Scotland. 2021. Reference Source

17. Generation Scotland: RuralCovidLife Survey General Report: Health and wellbeing of rural communities in Scotland. University of Edinburgh: Edinburgh. 2020.

18. Qualtrics. Provo, Utah, USA. 2020. Reference Source

19. McKinstry B, Sullivan FM, Vasishta S, et al.: Cohort profile: the Scottish Research register SHARE. A register of people interested in research participation linked to NHS data sets. BMJ Open. 2017; 7(2): e013351. PubMed Abstract | Publisher Full Text | Free Full Text

20. Huggins CF: Extended data for "RuralCovidLife: Study protocol and description of the data". 2021.

21. Kroenke K, Spitzer RL, Williams JB: The PHQ-9: validity of a brief depression severity measure. J Gen Intern Med. 2001; 16(9): 606-613. PubMed Abstract | Publisher Full Text | Free Full Text

22. Spitzer RL, Kroenke K, Williams JBW, et al.: A Brief Measure for Assessing Generalized Anxiety Disorder: The GAD-7. Arch Intern Med. 2006; 166(10): 1092-1097.

PubMed Abstract | Publisher Full Text

23. Scottish Government: Scottish household survey 2018: annual report. 2018.

24. Skerratt S, Meador E, Spencer M: National Rural Mental Health Survey Scotland: Report of Key Findings. Scotland's Rural College. 2017. Reference Source

25. Scottish Rural Action: Rural Communities Survey on COVID-19 - Response and Recovery. 2020.

26. Manea L, Gilbody S, McMillan D: Optimal cut-off score for diagnosing depression with the Patient Health Questionnaire (PHQ-9): a metaanalysis. CMAJ. 2012; 184(3): E191-E196. PubMed Abstract | Publisher Full Text | Free Full Text 


\section{Open Peer Review}

\section{Current Peer Review Status:}

\section{Version 1}

Reviewer Report 11 May 2022

https://doi.org/10.21956/wellcomeopenres.19155.r49908

(C) 2022 Hope $\mathbf{H}$. This is an open access peer review report distributed under the terms of the Creative Commons Attribution License, which permits unrestricted use, distribution, and reproduction in any medium, provided the original work is properly cited.

\section{Holly Hope}

Centre for Women's Mental Health, School of Health Sciences, University of Manchester, Manchester, UK

This is a really interesting piece of research that describes the impact of COVID on rural Scotland.

Here are my main thoughts, that need to be addressed prior to indexing:

\section{Introduction:}

Lacks a clear set of objectives as to what this paper does.

I do not see how this is a study protocol as it has no objectives, and the data is already collected.

\section{Results:}

Rural Scotland $\sim 900,000$ people, this sample is only $\sim 3000$, what measures were taken to address potential sources of selection bias, for example are any of the studies you recruited from use population based sampling?

Minor - some typos throughout, need another review and those removed, see ethic section for an example.

Results; "Following data cleaning, 1,270 participants were included, leaving 3,365 participants in the final dataset."

Should this read:

"Following data cleaning, 1,270 participants were excluded, leaving 3,365 participants in the final dataset."

\section{Discussion:}

"the study is not representative, with the majority of respondents being older and female. 
Participants were recruited through a mental health network, and a high proportion of participants scored above cut-off on the GAD-7 and PHQ-9. As such, participants with mental distress may be over-represented."

I would also suggest that the absence of rural communities from more deprived areas may also underestimate mental health risk.

At any time, 1 in 5 women and 1 in 8 men report a mental illness. apms-2014-full-rpt.pdf (publishing.service.gov.uk). There is considerable evidence that women's mental health was most affected by COVID restrictions. Please see Mental health before and during the COVID19 pandemic: a longitudinal probability sample survey of the UK population - PubMed (nih.gov) and Mental health responses to the COVID-19 pandemic: a latent class trajectory analysis using longitudinal UK data - PubMed (nih.gov).

Therefore, the overall rate of $\sim 1$ in 5 suggests that poor mental health has increased for either men or women, the discussion lacks any discussion for why that might be.

Have the authors considered weighting the GAD or PHQ estimates to give a more accurate overall estimate, or considered reporting scores for men and women (in age bands) separately, to give a more nuanced overview of mental health (and therefore a little more to discuss in the discussion)?

Why do we see higher rates of loneliness among young people in rural Scotland than older people - are these findings replicated in GAD and PHQ (I suspect they are) and does this have any policy implications?

The possibility of linkages to other datasets is useful, but it would be helpful for the reader if the authors could envisage the types of projects that are possible with this dataset and any planned future analyses in the discussion.

Is the rationale for creating the dataset(s) clearly described?

Yes

Are the protocols appropriate and is the work technically sound? Partly

Are sufficient details of methods and materials provided to allow replication by others? Partly

Are the datasets clearly presented in a useable and accessible format? Partly

Competing Interests: No competing interests were disclosed.

Reviewer Expertise: Psychiatric epidemiology, impact of COVID, behavioural science.

I confirm that I have read this submission and believe that I have an appropriate level of expertise to state that I do not consider it to be of an acceptable scientific standard, for reasons outlined above. 
Author Response 16 May 2022

\section{Charlotte Huggins}

Thank you for your thoughtful and detailed review. Please find below responses to your key points. These have been addressed as best as possible in the newest version of the manuscript.

Introduction:

- Lacks a clear set of objectives as to what this paper does.

- I do not see how this is a study protocol as it has no objectives, and the data is already collected.

We agree that the objective of the paper was not clearly enough stated in the previous draft, and this has caused some confusion. We would like to stress that this is a Wellcome Open Research 'Data Note'. This means it makes no interpretation of the collected data, and is instead intended only to describe the data resource to make it available for other research use.

To clarify this, we have changed the title of the paper to:

"RuralCovidLife: A new resource for the impact of the pandemic in rural Scotland"

We have also added a small line to the introduction, to clarify the purpose of the paper, see line 208 - 210:

"This is a data note intended to act as an information resource for researchers, and does not make any interpretations of the data or its implications."

Results:

- Rural Scotland 2900,000 people, this sample is only 3000, what measures were taken to address potential sources of selection bias, for example are any of the studies you recruited from use population based sampling?

Unfortunately, the data was collected through convenience sampling, due to the need for a quick turnaround to collect relevant data during the early phase of the pandemic while conducting the participatory element of the study. This limitation has been clarified in the methods section, see line 310 - 311:

"Recruitment was conducted through convenience sampling, so sample may not be representative."

- Minor - some typos throughout, need another review and those removed, see ethic section for an example.

Thank you, the paper has been carefully proofread to identify any outstanding issues, and these have been removed.

- Results; "Following data cleaning, 1,270 participants were included, leaving 3,365 participants in the final dataset."

Should this read:

"Following data cleaning, 1,270 participants were excluded, leaving 3,365 participants in the final dataset." 
Thank you for pointing out this error. This has been amended.

Discussion:

- "the study is not representative, with the majority of respondents being older and female. Participants were recruited through a mental health network, and a high proportion of participants scored above cut-off on the GAD-7 and PHQ-9. As such, participants with mental distress may be over-represented."

I would also suggest that the absence of rural communities from more deprived areas may also underestimate mental health risk.

Thank you for highlighting this, we have added a statement acknowledging these important issue, see line 466 - 467:

"Participants also tended to be from less deprived areas, so the impact of SES on mental health and COVID-19 impact may be underestimated"

- At any time, 1 in 5 women and 1 in 8 men report a mental illness. apms-2014-full-rpt.pdf (publishing.service.gov.uk). There is considerable evidence that women's mental health was most affected by COVID restrictions. Please see Mental health before and during the COVID-19 pandemic: a longitudinal probability sample survey of the UK population - PubMed (nih.gov) and Mental health responses to the COVID-19 pandemic: a latent class trajectory analysis using longitudinal UK data - PubMed (nih.gov).

Therefore, the overall rate of $\sim 1$ in 5 suggests that poor mental health has increased for either men or women, the discussion lacks any discussion for why that might be.

We agree this is an important issue. However, Wellcome Open Research Data Notes are not intended to give interpretations of data. It is instead intended as a relatively neutral description of the data and how it was collected, to inform future researchers using the resource. As such, we do not believe it is appropriate to make such assertions in this paper. This would be an interesting question for a researcher using the resource to investigate.

- Have the authors considered weighting the GAD or PHQ estimates to give a more accurate overall estimate, or considered reporting scores for men and women (in age bands) separately, to give a more nuanced overview of mental health (and therefore a little more to discuss in the discussion)?

- Why do we see higher rates of loneliness among young people in rural Scotland than older people - are these findings replicated in GAD and PHQ (I suspect they are) and does this have any policy implications?

We agree the above two points are important considerations that merit further careful discussion and analysis. However, the current submission is intended as a descriptive resource, rather than a scientific analysis of the data. We therefore believe such discussion and interpretation of the results would not be appropriate for a data note.

Competing Interests: No competing interests were disclosed. 